\title{
Allergy - hypersensitivity with hyperreactivity
}

\author{
Dmitry Novikov ${ }^{1}$ \\ ${ }^{1}$ Vitebsk State Order of Peoples' Friendship Medical University
}

August 25, 2020

\begin{abstract}
A definition of allergy is proposed, including its two stages: 1) hypersensitivity (without clinical manifestations) and 2) hyperreactivity with clinical signs. A specific immunologically mediated allergy depends on the presence of antibodies of different isotypes and immune T and B lymphocytes against an allergen. Nonspecific allergy is hypersensitivity and hyperreactivity of the immune system and any cells, as well as humoral systems, to the pathogen. Anergy is the second type of pathologically altered reduced reactivity that can be of allergen-specific (immune) and non-immune types.
\end{abstract}

\section{Allergy - pathologically altered body reactivity}

The term "allergy" proposed by C. Pirquet in 1906 denoted theacquired change in the specific reaction of the body to infectious antigens in tuberculosis and syphilis [1].

The classic nomenclature of 4 types of hypersensitivity was proposed by P.G. Gell and R.R. Coombs [2]. However, the term "hypersensitivity" began to replace "allergy". Therefore, the proposal of Ring J. et al. [3], Jutel M., Ring J. [4] to revise the nomenclature for allergy is very timely.

We propose for discussion the definitions of allergy, hypersensitivity and hyperreactivity that differ from the generally accepted ones.

The reactivity of an organism is a genetically determined aggregate local and/or general variant of response of its systems - immune, nervous, endocrine, integumentary tissues (skin, mucous membranes) and organs to a pathogen-irritant.

The problem of changing of reactivity to external stimuli has been of interest in medicine for many centuries. The norm of reactivityis the midline that distinguishes a diseased person from a healthy one. Changes in reactivity can be physiological andpathological (Fig. 1). Body reactions to any pathogens can benormergic, hyperergic, hypoergic andanergic. Their appearance depends on the genetic predisposition and pathogens. Allergy is usually associated only with increased reactivity. However, there is a decrease in reactivity: hypoergicand anergic response, which is often observed with immunodeficiencies, but also occurs in allergy. Immunodeficiencies are accompanied by genetically reduced reactivity and, apparently, should be a type of anergy.

Therefore, two main types of pathological reactivity should be distinguished: allergy with increased reactivity and anergy withreduced reactivity. C. Pirquet [1] considered allergy as an "altered" reaction of an organism, i.e. increased and reduced. However, allergists are not ready for such understanding of allergy, even if it is generally correct.

\section{Types of allergy}

According to WAO-EAACI consensus [5, 6], "hypersensitivity" dominates in the definition of allergy: allergy is a hypersensitivity reaction initiated by immunological mechanisms. EAACI also gives the following definition 
[5]: hypersensitivity causes objectively reproducible symptoms or signs that develop after exposure to a specific stimulus in doses to which normal subjects are tolerant. In these definitions, allergy is nominated as hypersensitivity, which is inaccurate because hypersensitivity is the first stage of an allergic reaction that ends with the release of mediators from the cells. They can be detected not only in vitro after incubation of sensitized basophils with allergen, but also in vivo even without allergy clinic signs [7, 8, 9]. Released mediators nonspecifically involve other leukocytes, which is usually called the "late phase" of the reaction. However, this is the initial stage of hyperreactivity, i.e. the second stage of allergy, in which all local cells and tissues are involved, which ultimately causes clinical symptoms (Fig. 2). We can assume that allergy is an increased response of a genetically predisposed organism to low doses of allergens (immune) or any pathogens - substances and physical factors (nonspecific) that do not cause such a reaction in the vast majority ([?]85\% or more) of healthy people.

Therefore, allergy includes two types of reactions that develop in succession in two stages - hypersensitivity , which is realized at the cellular-molecular level, ending with the release of mediators, and hyperreactivity , leading to the development of a local (hyperemia, edema, etc.) and general reaction involving various cells and tissue structures (Fig. 2).

Specific immune allergy develops as a result of an adaptive immune response with the formation of IgE, nonIgE antibodies and immune T and B lymphocytes (Fig. 3). EAACI divides IgE-dependent reactions into atopic and non-atopic. Atopy, as defined by EAACI [6], is a personal or familial tendency to produce $\operatorname{IgE}$ antibodies in response to low doses of allergens, usually proteins, and to develop typical symptoms such as asthma, rhinoconjunctivitis or eczema/dermatitis. However, there are other mechanisms of atopy: inducing effects of physical exercise in some patients, detection of nonspecific bronchial hyperreactivity in patients with allergic asthma, impaired skin barrier function in atopic patients, genetically determined dysfunction of the autonomic regulation of body systems - increased cholinergic reactivity and $\alpha$-adrenergic reactivity and decreased $\beta$-adrenoreactivity. Therefore, in atopy, along with IgE antibodies, there is nonspecific hypersensitivity and hyperreactivity.

In addition, granulocytes (neutrophils, etc.) and even platelets can participate in IgE-mediated reactions. Atopic neutrophils have FceRI [10], FceRII (CD23), and galectin-3 receptors [11]. They are also present on all leukocytes and platelets that bind $\operatorname{IgE}$ and can interact with allergens (Fig. 3).

Other types of non-IgE allergy and, accordingly, immune hypersensitivity are carried out by IgG, possibly IgM and IgA antibodies with the participation of granulocytes, as well as immune $\mathrm{T}$ and B lymphocytes (see Fig. 3). Based on these types of hypersensitivity and subsequent hyperreactivity, immediate allergic reactions arise (cytotoxic, immunocomplex reactions), as well as delayed type allergy mediated by immune $\mathrm{T}$ cells, which includes 4 subtypes of hypersensitivity [4]. However, each of these subtypes of allergy is accompanied by hyperreactivity with clinical signs. At the same time, hypersensitivity, i.e. sensitization of cells, determined in vitro, is not called allergy, and in vivo with low doses of allergens it can occur without clinical manifestations $[8,9]$.

Nonspecific immune allergy (Fig. 2) is realized after nonspecific activation by pathogens of humoral factors and cells of innate and adaptive immunity. Often it is dose-dependent and is observed only at high doses of pathogen. Its example is non-immune complement activation via the alternative or lectin pathway with the release of anaphylatoxins and clinical presentation of anaphylaxis (shock). It can occur after intravenous administration of more than 50-100 ml of solutions of drugs or blood substitutes. Activation of lymphocytes by mitogens and superantigens, degranulation of basophils, eosinophils and neutrophils under the influence of nonspecific agents and physical factors (cold, heat, vibration, sunlight, physical activity) can induce nonspecific hypersensitivity, leading to hyperreactivity and the development of allergic diseases.

Nonspecific non-immune allergy (Fig. 3) is an increased reaction of non-immune cells to the action of a pathogen to which there is hypersensitivity, which causes the release of mediators and cytokines from them. They engage other cells in response and induce hyperreactivity, which leads to clinical symptoms of allergy.

Hypersensitivity is an increased reaction of cells or humoral systems of a predisposed organism to an aller- 
gen or nonspecific pathogen that act on cell receptors or key molecules and cause the release of intracellular products - cytokines, mediators, enzymes, or induce cascade activation of humoral systems (for example, complement). The resulting hypersensitivity products activate other cells and tissues, involving them in the reaction, which leads to the development of hyperreactivity reaction of tissues, mucous membranes, smooth muscles, nervous and endocrine systems.

Both immune and non-immune hypersensitivity depend on the number and affinity of cell receptors interacting with a particular pathogen, and the activity of structures that receive signals of this interaction and lead to the release of mediators, cytokines, enzymes from cells, or to cascade activation of humoral factors of the immune and neuroendocrine systems.

Hyperreactivity is an increased response of various cells, systems and organs of the body to mediators and cytokines released during the hypersensitivity reaction and causing clinical symptoms or signs to low or high doses of the pathogen that do not cause such a reaction in most people. It is always nonspecific but can differ in induced clinical syndromes when it develops after a specific immune or non-immune hypersensitivity due to differences in the spectrum of mediators. Hyperreactivity as body's aggregate response to a hypersensitivity reaction depends on different systems, cells, humoral factors, which are nonspecifically involved in a pathologically increased response accompanied by damage to cells, tissues and organs.

Using skin tests with an allergen, it is possible to determine the minimum dose of an allergen that causes allergic skin reaction, which includes both hypersensitivity of basophils and hyperreactivity of cells and structures of skin (blood vessels, epithelium, etc.) to mediators released by them. However, the assessment of skin tests for an allergen is carried out according to the degree of hyperreactivity (edema, hyperemia).

Bronchial hyperreactivity can be inhibited using $\beta_{2}$ receptor agonists or glucocorticosteroids. However, their hypersensitivity persists, which is detected after the abolition of these drugs.

Nonspecific hyperreactivity after nonspecific hypersensitivity, as well as after specific allergen-specific hypersensitivity, leads to the induction of clinical symptoms from the skin (hyperemia, edema, etc.), nasal mucous membranes, bronchi, intestines, etc. (hypersecretion, edema, smooth muscle contraction) by nonspecific stimuli: smoke, cold air and various irritants. For example, in nasal nonspecific hyperreactivity various symptoms are induced: rhinorrhea, edema, obstruction of the nasal passages with external stimuli cigarette smoke, cold and other irritants. However, they cause similar types of hyperreactivity and clinics in allergic and non-allergic rhinitis [11].

\section{Dose-dependence of hypersensitivity and hyperreactivity}

These two stages of allergy are related by the dose-dependent effect of allergens and non-specific inducers. However, hyperreactivity is completely dependent on the degree, intensity of hypersensitivity, therefore, the dose of an allergen or inducer determines only the degree of specific or nonspecific hypersensitivity. The need for high doses of inducers for nonspecific types of allergy is due to the relatively low concentration, expression of cell receptors or activating molecules of humoral factors that respond to them (for example, complement).

Hypersensitivity of cells is easily detected in vitro by their release of mediators, cytokines and enzymes. All leukocytes, if they are sensitized actively (lymphocytes) or passively (with antibodies - granulocytes, platelets) respond to the allergen by the release of these substances and degranulation to low doses of allergens, as well as by change in phenotype, expression of receptors and CD antigens. In some patients, nonspecific inducers, for example drugs with nonspecific hypersensitivity to them, also provoke such a response $[12,13]$.

The in vivo response to allergens and nonspecific inducers is assessed by the level of hyperreactivity: by the degree of skin reaction (hyperemia, edema, etc.), or reaction of bronchi (bronchospasm), as well as by the clinical symptoms of allergy that it causes (urticaria, rhinorrhea, etc.) It was possible to evaluate hypersensitivity in vivo without hyperreactivity and clinical symptoms after developing in our clinic [7, $8,9]$ low-dose allergen-specific nasal and oral-pharyngeal tests that do not cause clinical symptoms, but 
are recorded by an increase in the level of allergy biomarkers in nasal secretions and oral fluid. Doses of allergens in these tests were reduced by 20-200 times compared with those used in conventional provocative tests, which induce clinical symptoms.

Allergy is a specific immune and nonspecific non-immune reaction of hypersensitivity of cells and humoral systems of a genophenotypically predisposed organism to allergens, as well as to various pathogens, that cause the release of mediators that induce increased nonspecific reactions of other cells and systems, which leads to the development of hyperreactivity, damaging tissue.

\section{Role of other systems and cells in allergy}

Nonspecific allergy (nonspecific hypersensitivity and hyperreactivity) may occur due to the initial genetically and/or phenotypically altered reaction of various cells and humoral systems.

In allergic reactions, leukotrienes are involved. Under the influence of various pathogens on leukocytes, phospholipase A is activated, which involves in the metabolism arachidonic acid from phospholipids of cell membranes. The products of its metabolism - eicosanoids - are involved in the formation of leukotrienes andprostaglandins, inflammatory mediators that cause the development of hyperreactivity. LT-B4 is a strong chemoattractant for neutrophils and eosinophils, it stimulates inflammation, LT-C4 enhances edema and histamine release, prostaglandin PGD2 causes smooth muscle contraction.

Immunoneuroendocrine interactions determine overall body homeostasis and its reactivity. The cytokines of the immune system affect the functions of neurons, synapses, cells of the endocrine system. On the other hand, neurotransmitters and hormones alter the responses of the immune system.

Dysmetabolic - a dysregulatory nonspecific variant of allergy is characterized by the fact that with genetically determined metabolic abnormalities, pathogens and provoking factors induce the release of mediators and cytokines. The receptor-mediator imbalance in the regulation of the cumulative effects of the immune, nervous and endocrine systems on various impacts usually determines the development of nonspecific allergy.

An imbalance in the regulation of the nervous system can cause the occurrence of nonspecific allergy. The sympathetic nervous system through $\beta$-adrenergic receptors $\left(\beta_{1}-\right.$ constriction, $\beta_{2}$ - dilatation), and the parasympathetic through $\mathrm{M}$-cholinergic receptors (constriction) regulate smooth muscle tone and the release of mediators from cells. The predominance of the activity of the parasympathetic nervous system over the sympathetic, decrease in the activity and number of $\beta_{2}$ receptors on the cells during stimulation by nonspecific agents leads to the predominance of M-cholinergic receptors and inflammatory mediators. The increased activity of non-cholinergic excitatory nerves causes the release of neuropeptides: vasoactive intestinal peptide (VIP) - substance P, neurokinin A, calcitonin gene-related peptide (CGRP), inducing edema, hypersecretion, bronchospasm, skin reactions.

Dysfunctions of the endocrine system (thyroid hormones, cortisol, estrogens, etc.) lead to a change in reactivity. Often there aredishormonal allergy variants caused by impairments in the relationship of the hypothalamus-pituitary-adrenal cortex, which leads to primary or secondary adrenal insufficiency and steroid dependence in the treatment of pathology, for example, bronchial asthma, chronic urticaria. In cases of impaired ovarian function and menstrual cycle, menopause, an imbalance of estrogen and progesterone develops, which is the cause of urticaria and asthma.

Any cells can participate in the development of allergy (Fig. 3).Nonspecific allergic reactions, apparently, can be triggered and carried out by those cells that have receptors that bind the pathogen, or signal structures that respond to the physical factor (light, cold). However, their response should be increased. Epithelial and endothelial cells, fibroblasts and other cells are capable of secreting cytokines, enzymes and other molecules under the influence of pathogens and, if their response is abnormal, increased, this can lead to the development of allergy (Fig. 2).

Epithelium stimulated by pathogens secretes IL-25, IL-33 and thymic stromal lymphopoietin (TSLP). These 
cytokines mobilize the migration of dendritic cells to the lymph nodes, where they activate naïve T lymphocytes in Th2, which are involved in allergy.

Smooth muscle cells have receptors for mediators and cytokines of allergic reaction and their contraction is an important sign of allergic diseases (asthma, rhinitis, intestinal allergy).

Vascular endothelium responds to hypersensitivity mediators with an increase in permeability, which leads to edema, skin hyperemia, subendothelial leukocyte infiltration. Endothelial cells secrete a number of cytokines.

Fibroblasts, when activated by neurotransmitters and cytokines, secrete the precursors of collagen and elastin proteins, as well as mucopolysaccharides, and cytokines.

Therefore, allergy as pathologically altered reactivity may include immune and non-immune mechanisms.

\section{Anergy}

Anergy is a hyporeactive type of reactivity usually observed to infectious antigens. It can be specific (immune) and nonspecific - non-immune. With it, there is no hyperreactivity, but there is tolerance to an allergen or immunosuppression after the use of immunosuppressants.

Allergen-specific immuno-mediated anergy is due to hypo- or areactivity to allergens in a patient with allergy and can be immunosuppressive or immunoregulatory. After successful allergen-specific immunotherapy (ASIT), patients remain allergic, but they develop anergy to the allergen. Such anergy differs from pathological anergy, in which there is no response to stimulation with an antigen (infectious or non-infectious) due to a genetically determined lack of response or due to immunosuppression.

Pathological anergy can cause immunodeficiency diseases and infections.

On the other hand, "immunity" which is unsusceptibility to infection, can be also essentially considered anergy. Additional data on the commonality and differences of these concepts is needed.

The anergy of non-immune cells, especially epithelial cells releasing defensins and antibacterial peptides, often leads to immune deficiency and the development of infections.

\section{Conclusion}

Thus, our proposals for nomenclature and classification of allergy include the following points:

1. Pathologically altered body reactivity can be of two types: increased, hyperreactive - allergy, and reduced - anergy.

2. Allergy includes two reaction stages: 1) hypersensitivity (without clinical manifestations) and 2) hyperreactivity with clinical symptoms.

3. A specific immunologically mediated allergy depends on the presence of antibodies of different isotypes and immune $\mathrm{T}$ and $\mathrm{B}$ lymphocytes to the allergen.

4. Nonspecific allergy is hypersensitivity and hyperreactivity of the immune system and any cells, as well as humoral systems, to the pathogen.

5. Anergy is the second type of pathologically altered reduced reactivity, and it can be allergen-specific (immune) and non-immune. A kind of antigen-specific anergy to an infection agent may be immunity itself, although at first glance it seems absurd. But normergia is a normal immune response to infection, while hyperergic reponse is a disease.

\section{References}

1. Von Pirquet C. Allergie. Munch Med Wochenschr. 1906; 30: 1457-1458.

2. Gell PGH., Coombs RRA. Clinical Aspects of Immunology. $2^{\text {nd }}$ ed. Oxford: Blackwell. 1968: 575-596.

3. Ring J, Jutel M, Papadopoulos N et al. Provocative proposal for a revised nomenclature for allergy and other hypersensitivity diseases. Allergy; 2018, 73: 1939-1940. 
4. Jutel M, Ring J. Revised nomenclature for allergic diseases. EAACI White Paper; 2018: 111-113.

5. Johansson SG, Hourihane JO. A revised nomenclature for allergy - An EAACI posit ion statement from the EAACI nomenclature task force. Allergy; 2001; Vol. 56: 813-824.

6. Johanson SGO., Bieber T, Dahl R et al. Revised nomenclature for allergy for global use: Report of the Nomenclature Review Committee of the World Allergy Organization, October 2003. Allergy and Clin. Immunol.; 2004; vol. 113, 5: 832-836.

7. Shchurok IN. Biomarkers of oral fluid after oral-pharyngeal provocative test with allergen for diagnosis of atopic bronchial asthma. Immunopathology, allergology, infectology. 2018; 4: 82-86. DOI: 10.14427/jipai.2018.4.82.

8. Shchurok IN. Increase in the enzymatic activity of nasal lavage and oral fluid in allergic rhinitis under the influence of low allergen doses. Immunopathology, allergology, infectology 2019; 1: 89-94. DOI: 10.14427/jipai.2019.1.89.

9. Shchurok IN. The identification of the mediators for specific and nonspecific upper respiratory tract hypersensitivity diagnosis by the provocative tests. Allergy. N J USA: WILEY, 2018; T. 73: 400.

10. Gounni AS, Lamkhioued B, Koussih L et al. Human neutrophils express the high affinity receptor for IgE (FceRI): role in asthma. FASEB J. 2001; 5: 940-949.

11. Truong MJ, Gruart V, Kusnierz JP et al. Human neutrophils express immunoglobulin E (IgE)-binding proteins $(\mathrm{Mac} 2 / \varepsilon \mathrm{BP})$ of the S-type lectin family: role in IgE-dependent activation. J. Exp. Med. 1993; 117: $243-248$.

12. Segboer CL, Holland CT, Reinartz SM et al. Nasal hyper-reactivity is a common feature in both allergic and nonallergic rhinitis. Allergy; 2003; vol. 68, Issue 11: 1427-1434.

13. Rozieres $\mathrm{A}$ et al. Role of $\mathrm{T}$ cell in nonimmediate allergic drug reactions. Curr. Opin. Allergy Clin. Immunol.; 2009; 9: 305-310.

14. Beeler A et al. CD69 upregulation on T cells as an in vitro marker for delayed-type drug hypersensitivity. Allergy; 2008; 63: 181-188.

Figure 1. Types of body reactivity

Figure 2. Type I (immediate) allergy

ECFs - eosinophil chemotactic factors, PAF - platelet-activating factor, NCFs - neutrophil chemotactic factors, PDGF - platelet-derived growth factor

Figure 3. Types of allergy and structures realizing it

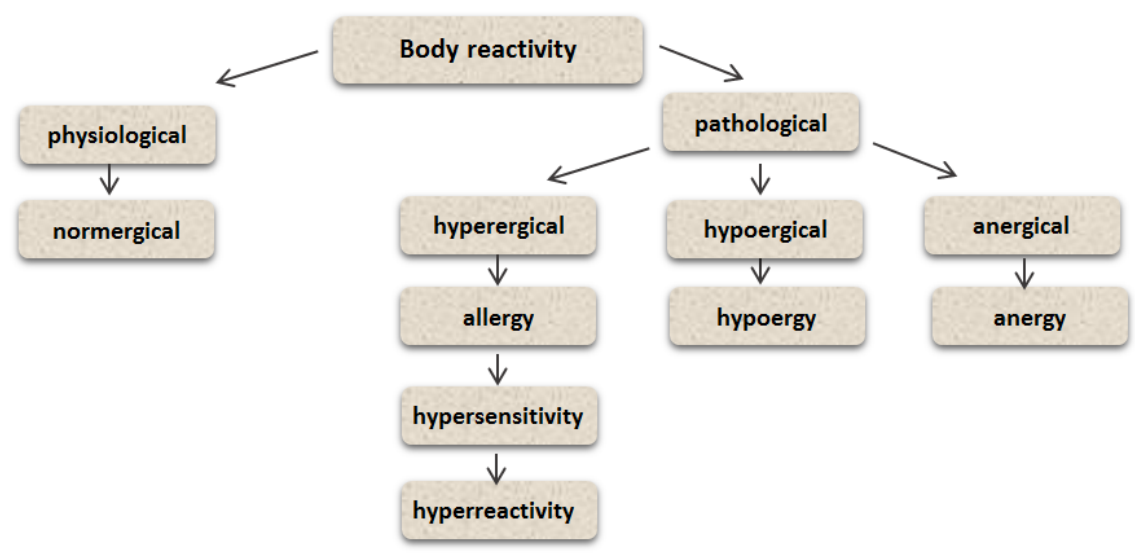



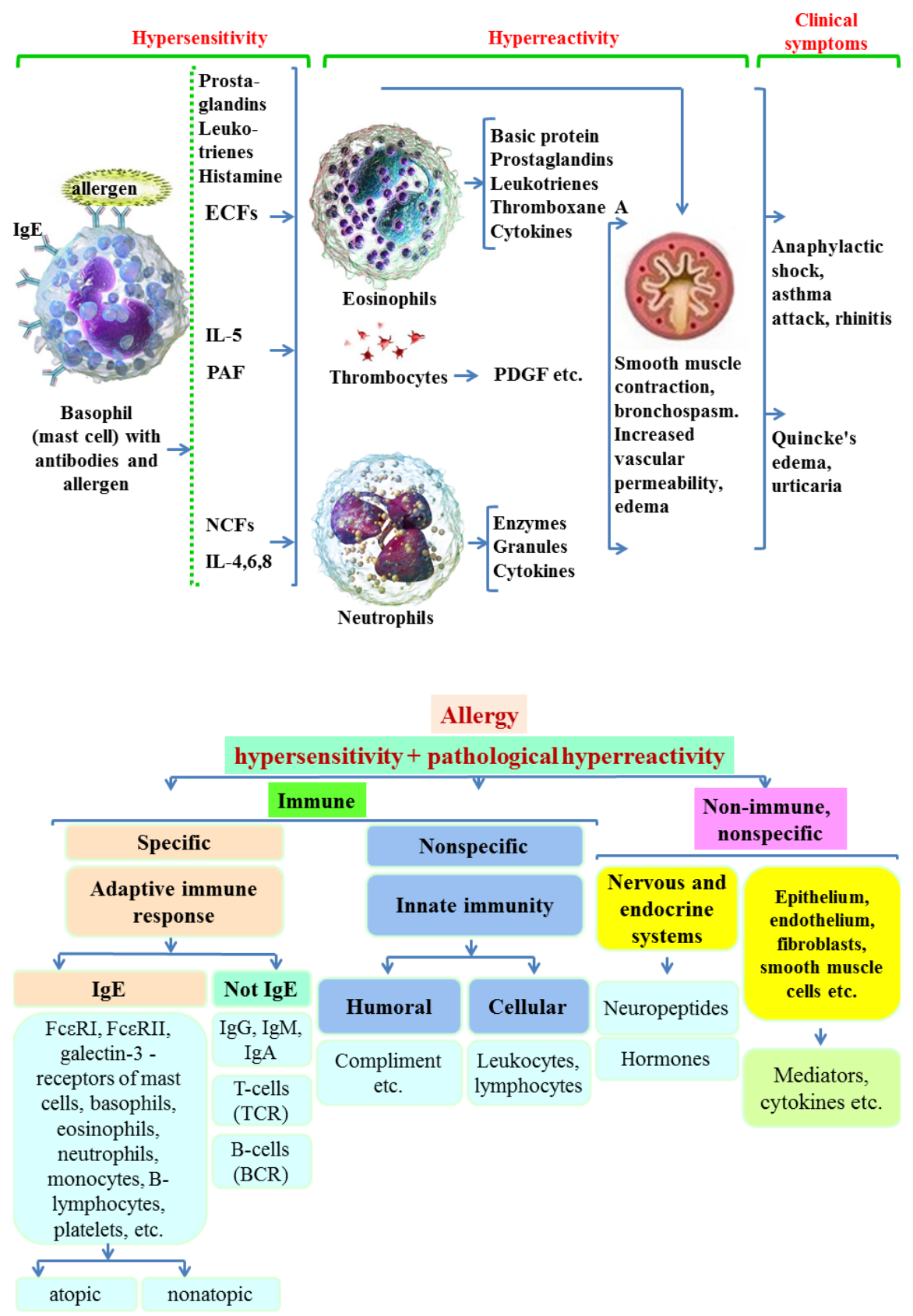